\title{
The New Science of Attention Deficit Hyperactivity Disorder: News from the Cutting Edge of Research Science
}

\section{Elizabeth Gilley*}

Research Review Analyst, Founder The Elle Foundation, West Palm Beach, FL, 33401, USA

"Corresponding Author: Dr. Elizabeth Gilley, Research Review Analyst, Founder The Elle Foundation, West Palm Beach, FL, 33401, USA, Tel: 1-561-229-6382; E-mail: gilley.elizabeth@yahoo.com

Received: 14 May 2018; Accepted: 22 May 2018; Published: 30 May 2018

\begin{abstract}
The evolving science of Attention Deficit Hyperactivity Disorder garners insight from many sciences for enlarged perspective, expanding theoretical understanding, and broadening treatment applications. Comorbidity and ADHD's shared characteristics with other disorders have made diagnosis difficult, especially when professional trends base diagnosis upon a cluster of symptoms. Neuroscience is now pinpointing energetic signatures of the disease's electromagnetic expression. In addition to traditional pharmacological and behavioral therapeutic treatments, neuro-therapy is being prescribed in the form of neurofeedback.
\end{abstract}

The amount of research on the global spectrum is extremely vast, far reaching, crossing over the limitations of former boundaries, and expanding exponentially. It is a daunting task for those in the practice of medicine to keep abreast of the latest research in the field of one's own specialty, much less to be informed of the advancements in inter-related or cross over fields. Typically, research science is thirty to fifty years ahead to what is available to the public. For example, based upon the past fifty years of research, a new disease has come to the forefront of the research arena, Reward Deficiency Syndrome (RDS). Addiction is one of its behavioral expressions. This is huge. Addiction is no longer viewed as the disease. It is the symptom. ADHD is closely associated with RDS and is viewed as a precursor to addiction. On the post doctoral level of research science, for those in the know, there is no debate. However, unfortunately, many in the practice of medicine are uninformed, and still using last century's ADHD and addiction conceptual criteria.

Keywords: Attention Deficit Hyperactivity Disorder; Reward Deficiency Syndrome; Neuroscience 


\section{New Science of Attention Deficit Hyperactivity Disorder}

The American Psychological Association's Diagnostic and Statistical Manual of Mental Disorders, $5^{\text {th }}$ Edition, states ADHD is a "neuro-developmental disorder defined by impairing levels of inattention, disorganization, and/or hyperactivity-impulsivity" [1]. The DSM-5 outlines specific presentations of ADHD as 1) predominantly inattentive; 2) predominantly hyperactive/impulsive; 3) combined presentation; 4) other specified ADHD activity; and 5) unspecified ADHD disorder. The DSM-5 suggests that no specific genes are causal and that there are no biological markers for diagnosis. Scientifically speaking that is absolutely correct. Genes are not causal. They are however, correlational. It is the interaction of genetic and epigenetic pressures which determines outcome.

Research review of clinical and molecular genetics provides detailed lists of genes that neuroscientists have found which contribute to ADHD. Dr. David Comings, a psychiatrist, psychologist, and neuroscientist, defines ADHD as a "polygenic disorder due to the additive effect of genes affecting dopamine, norepinephrine, serotonin, GABA, and other neurotransmitters" [2]. In addition to the peer reviewed publications of Dr. Comings, Dr. Florence Levy has also made substantial contribution to the knowledge base of the genetics of ADHD [3].

Dr. Kenneth Blum, well known for pioneering the term Reward Deficiency Syndrome, and Dr. Kevin Miller believe that Attention Deficit Disorder and the addicted brain are on overload, that it is a genetic defect which causes neurological imbalance, with some brain centers being hyperactive, while others have decreased activity [4]. Dr. Kenneth Blum views ADD/ADHD as a precursor to addictive expression, with its roots closely associated with the D2 receptor and the A1 Allele [5].

Neuroscientists have shown that ADHD has bio-genetic components, due to genetic polymorphism, and it is closely associated with Reward Deficiency Syndrome, dopamine and serotonin system imbalance, which contribute to future addictive expression [4]. "Dopamine gene abnormalities are common in ADHD and medications that increase dopamine availability in the brain, such as stimulants like Ritalin or Adderall, are used to treat it. ADHD is associated with low activity in the prefrontal cortex. Low dopamine levels are also associated with low PFC. When the Ventral Tagmental Area (VTA) does not produce enough dopamine, it cannot drive the Basil Ganglia Prefrontal Cortex circuit into appropriate action" [6].

It is crucial for the field of Psychiatry to understand that the old paradigm of dopamine antagonist therapy for addiction has not worked, although it may appear to be the logical treatment response for addictive behavioral expression when viewed through old conceptual frames. Putting on a new pair of glasses, viewing ADHD as a precursor to and addiction as symptom of Reward Deficiency Syndrome (RDS) will help everyone get on the same page. The Blum team of associates suggests that the establishment open their minds to trying a new paradigm of dopamine agonist therapy in the treatment of RDS related impulsive, compulsive, and addictive behavioral expression. Increasing dopamine availability works for both ADHD and addiction.

While biological markers may not have been identified, yet, genetic and energetic markers have been identified. ADHD has an energy signature. Several in fact. Various forms of brain imaging like single photon emissions computed tomography (SPECT) over several brain regions provide neurological evidence of blood flow, whether 
there is too much activity or too little $[2,7]$. SPECT scans provide a 3 dimensional visual map of the degree of health of and/or trauma to the brain. Brain trauma can also cause symptoms similar to ADHD.

Some individuals have more than one type of ADD, depending upon their genetic vulnerability. Dr. Daniel Amen's current research supports seven distinct types of ADD/ADHD [8]. The spectrum of ADHD includes variations, different types of ADHD which include: 1) Classical ADD: 2) Inattentive ADD: 3) Over-focused ADD; 4) Temporal Lobe ADD: 5) Limbic ADD; 6) Ring of Fire ADD; and 7) Anxious ADD [8]. Each type corresponds to a specific brain center in which neurotransmission is either firing too fast or too slowly. Naturally each type requires a specific treatment. SPECT imaging can recognize problems in brain centers such as the Anterior Cingulate Gyrus, the Deep Limbic System, the Basil Ganglia, Temporal Lobes, Cerebellum, and Ventral Tagmental Area. Not knowing the exact area or reason for malfunction can lead to problems of interpretation.

For example, type 3, Over-focused ADD is characterized by "excessive activity in the anterior cingulate gyrus" [8]. While the SPECT may not reveal what is causing the malfunction, it does help to focus neurofeedback treatment [9]. In this example, training consists of focusing upon enhancing alpha activity. Different methods of neurofeedback have been established to treat the various brain centers that are showing excessive or decreased brain activity. However, some areas are too deep to penetrate. Often a combination of several types of neurofeedback are utilized to help the patient learn to tune in, become aware, and increase or decrease various brain wave patterns [10]. Another example would be ADHD patients who daydream excessively, showing an abundance of theta waves. These individuals can be taught how to focus on the beta wavelength.

Typical ADHD treatment prescribes psychostimulant pharmacotherapy, usually in the form of Adderall or Ritalin, combined with behavioral therapy. Fortunately, many advances have been made in neuro-pharmacology. New medicines have benefit for the now known 7 different types of ADHD. There are many considerations to be evaluated in the development of a treatment plan, including: 1) the potential long-term harmful effects of traditional stimulant therapy [11, 12]; 2) proclivity of genetic addictive predisposition or more accurately that of Reward Deficiency Syndrome; and 3) the possibility of pharmacologically induced neuro-adaptation [13].

While the APA may be correct in saying there are no biological markers for diagnosis, there are many biological components which need assessing. Dr. Amen lists: "Brain health, physical health, nutrition, exercise, sleep, hydration, hormones, blood sugar level, supplements, genetics, toxins, infections, physical illness, medication, trauma, injuries, and allergies" [8]. In addition to biological assessment, the patient also needs to be assessed psychologically, socially, and spiritually.

Dr. Amen has devised a comprehensive assessment Brain Type Test to be used in conjuncture with brain imaging to improve diagnostic criteria [14]. The test includes biological, psychological, social and spiritual paradigms to assess the extent of severity and damaging effects from ADD/ADHD, as it influences and effects all aspects of an individual $[8,14]$. Amen devises personal holistic treatment plans, tailored to the individual, which crosses over the boundaries of his specialized field of medicine, including application from other sciences. 
Many wellness applications, such as yoga, and mindfulness mediation $[15,16]$, can also help one to overcome the disorganization and lack of hyper focus associated with many types of ADD. Cognitive-behavioral psychotherapy helps the sufferer to learn adaptive skills to cope and overcome hardship. Applications from positive psychology which facilitate the enhancement of self-esteem, and improved life style choices, are often utilized in brain wellness regimes. Clients learn to manage their illness, and increase the likelihood of success and thriving.

Natural supplements have positive benefit for many brain centers. "GABA, an amino acid, can be used as a supplement to induce relaxation and offset stress. Vitamin B6 and L Glutamine support enzymes in producing GABA. Both Kava Kava and Valerian help calm anxiety and produce sleep. DI-Phenylalamine, increased protein intake, and Acetyl- L- Carnitine, and Omega-3 fatty acid supplements help boost brain efficiency” [6]. Dr. Kenneth Blum and Dr. John Giordano have patented NAT-therapy, neutraceutical blocks of amino acids to rebuild and enhance brain function [17].

A healthy diet can also help. Some types of ADHD respond to increased proteins while another may benefit from increased complex carbohydrates. The elimination of caffeine, sugar, and nicotine is recommended. Eliminating environmental toxins, as well as insecticides sprayed on food sources, help the brain to heal itself [14]. Increased exercise and sufficient sleep help to lessen ADHD expression. Educational awareness of electromagnetic pollution and its unsettling frenetic effects upon the mind, may help inspire discipline in turning off the television, and decreasing the amount of time spent using cell phones and playing video games.

ADHD is growing in the world population. As more countries adopt the American Psychological Association's diagnostic criteria, in diagnosing ADHD in children [18], it has been suggested that Big Pharma is "selling Attention Deficit Hyperactivity Disorder" [19]. Gratefully there are occasions when pharmacology is a bridge to healing. However, I am sure we all must have some ethical concerns about potential lies of omission, regarding potential harm, and /or the overprescribing of psychostimulants for developing brains. Are we to believe that there is really some magic healing pill that does not poison some aspect of the body's intricate balancing systems? Let us address the cause, not just the symptoms.

As both heredity and environmental factors influence the expression of ADHD, we must address life style choices, parenting skills, self-management, poor diets, environmental toxins, and an increasingly distractive culture. All of the above contribute to the poisoning of the brain, and subsequently, the mind. Let us not also poison the next generation with labels, and legalized speed, unnecessarily. Let us practice prevention and seek natural, holistic healing modalities. Let us teach patience, discipline and moderation, and lead by example. Let us seek the greater truth which honors all perspective.

As the masters of our own destiny, we must choose well. We can all do better. In the research arena, we need higher scientific standards. Newtonian physics is outdated, fractured, separatist, and inaccurate. We must rise to the occasion and embrace quantum physics. We can no longer afford to pretend that environmental and experiential influence does not matter. Need we be reminded that it is the field which determines the expression of the particle? Environmental and/or epigenetic pressure can quite literally rewrite or transcribe DNA. RNA is more potent than 
DNA in inducing evolutionary change. So let us turn our focus to environmental components. Let us focus upon an energy world, and seek energy medicine solutions.

On the neuro-therapeutic horizon, the new field of psycho-electro-magnetics Gilley [20] promises hope for future application from the realm of physics and healing frequencies, which may help individuals learn to realign and/or

fine tune their own energy fields, and protect themselves from electro-magnetic pollution. Anytime neuro-imbalance can be corrected, and stabilized, without poisoning some aspect of the brain's interactive neurological balancing system, it's a WIN. If at the same time, the treatment modality helps the patient acquire mental management skills, which may lead to mental mastery over his or her neuro handicap, it is a WIN WIN

It takes time for advancements from the cutting edge of science to trickle down even in the medical profession, much longer to the mainstream population. How do we speed this up so that doctors have the most up to date, current information and the population the opportunity to receive the best possible care? How do we bridge the distance between enhanced theoretical perspective and treatment application from the cutting edge of research science and prescribed standard treatment protocol as outlined by the DSM? By publishing research findings, research review and broadcasting news from the cutting edge of science, OFTEN! Help stop the generational cycle of Reward Deficiency Syndrome, ADHD and Addiction.

\section{References}

1. American Psychiatric Association. Diagnostic and Statistical Manual of Mental Disorders, 5th Edition. American Psychiatric Publishing (2013).

2. Comings D. Clinical and molecular genetics of ADHD and Tourette Syndrome. Annals of the New York Academy of Sciences, 931, Adult Attention Deficit Disorder: Brain mechanisms and life outcomes (2001): $50-83$.

3. Levy F, Hay D, Bennett K. Genetics of Attention Deficit Hyperactivity Disorder: A current review and future prospects. International Journal of Disability, Development and Education 539 (2006): 5-20.

4. Miller D, Blum K. Overload: Attention Deficit Disorder and the Addictive Brain. Miller Associates (2002).

5. Blum K, Braverman E, Chen A, et al. Dopamine D2 receptor Taq A1 Allele predicts treatment compliance of LG839 I s subset analysis of pilot study in the Netherlands. Gene Therapy and Molecular Biology 12 (2008): 129-140.

6. Amen D. Magnificent Mind at any age: Natural ways to unleash the brain's maximum potential: Treat anxiety, depression, memory problems, ADD, and insomnia. New York, NY: Three Rivers Press (2008).

7. Baughman F. The ADHD Fraud: Hoe psychiatry makes "patients" of normal children. Oxford, England: Trafford (2006).

8. Amen D. Healing ADD: The breakthrough program that allows you to see and heal the types of ADD, Revised Edition. Berkley (2013).

9. Lubar JF, Lubar JO. Neurofeedback Assessment and Treatment for Attention Deficit/Hyperactivity Disorders (ADD?HD). In Introduction to Quantitative EEG and Neurotherapy, edited by J. R. Evans and A. Abarbanel (1999): 103-143. 
10. Lubar J. Discourse on development of EEG diagnostics and biofeedback for AttentionDeficit/Hyperactivity Disorders (1991).

11. Mannuzza S, Klein R, Moulton J. Does stimulant treatment place children at risk for adult substance abuse? A controlled, prospect, follow-up study. Journal of Child and Adolescent Psychophar 13 (2004): 73-82.

12. Faccio E, Belloni E, Costa N. The use of the ADHD diagnostic label: What implications exist for children and their families? Procedia-Social and Behavioral Sciences 122 (2014): 506-509.

13. Leo J. Attention Deficit Disorder: Good Science or good marketing? Skeptic 8 (2000): 29-37.

14. Amen D. Healing ADD Brain Type Test. Amen Clinics (2014).

15. Van der Oord S, Bogels S, Peijnenburg D. The effectiveness of mindfulness training for children with ADHD and mindful parenting for their parents. Journals of Child and Family Studies 21 (2012): 139-147.

16. Singh N, Singh A, Lancioni G, et al. Mindfulness training for parents and their children with ADHD increases the children's compliance. Journal of Child and Family Studies 19 (2010): 157-166.

17. Blum K, Braverman E, Carbajal J, et al. Hypothesizing synergy between acupuncture/auriotherapy and natural activation of mesolimbic dopaminergic pathways: Putative natural treatment modalities for the reduction of drug hunger and relapse. Integrative Omics and Applied Biotechnology Letters 1 (2011): 1-14.

18. Conrad P, Bergey M. The impending globalization of ADHD: Notes on the expansion and growth of a medical disorder. Social Science and Medicine 122 (2014): 31-43.

19. Swartz A. The selling of Attention Deficit Disorder. New York Times (2013).

20. Gilley ED. Quantitative Research Study Design Prospectus: Psycho-Electro-Magnetic Light Therapy and Healing Frequency. West Palm Beach, FL: The Elle Foundation (2016).

Citation: Elizabeth Gilley. The New Science of Attention Deficit Hyperactivity Disorder: News from the Cutting Edge of Research Science. Journal of Psychiatry and Psychiatric Disorders 2 (2018): 71-76.

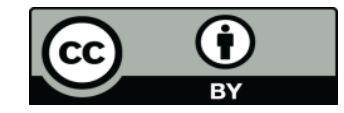

This article is an open access article distributed under the terms and conditions of the $\underline{\text { Creative Commons Attribution (CC-BY) license 4.0 }}$ 